Research article

\title{
Increased LncRNA PVT-1 is associated with tumor proliferation and predicts poor prognosis in cervical cancer
}

\author{
Yun-xiao Zhou ${ }^{\text {a*, }}$, Li-wei Mao ${ }^{\text {b }}$, Yan-li Wang ${ }^{c}$, Li-qun Xia a , Wei Zhao ${ }^{\text {a }}$ \\ ${ }^{a}$ Department of gynaecology, the First Affiliated Hospital of Zhejiang University Medical College; ${ }^{b}$ Department of Obstetrics and gynae- \\ cology, Jingning County People's hospital, Zhejiang; ' Department of Pathology, the First Affiliated Hospital of Zhejiang University Medi- \\ cal College
}

\begin{abstract}
Background: Cervical cancer is estimated to be the second most common cancer among women worldwide. The discovery of new and effective therapeutic strategies targeting cervical cancer is urgently needed. Upregulation of IncRNA PVT-1 and its biological significance in several cancers has been studied. However, its role in cervical cancer remains poorly understood. This study was designed to investigate the expression, clinical significance, and biological role of PVT-1 in cervical cancer.

Methods: Expression of PVT-1was analyzed in the sera of 120 cervical cancer patients and 118 normal control women by quantitative real-time PCR (qRT-PCR). The associations between PVT-1 and clinicopathological characteristics, as well as the survival probability of patients, were analyzed by statistical analysis. pcDNA3.1 and siRNAs were used to modulate PVT-1 expression in Hela and C33A cells. In vitro assays were performed to further explore the biological functions of PVT-1 in cervical cancer cells.

Results: The qRT-PCR results revealed high levels of expression of PVT-1 in cervical cancer patients, and PVT-1 was significantly higher in patients at advanced tumor stages and in patients whose tumor size was $\geq 3 \mathrm{~cm}$ and whose lymphatic node metastasis was positive. In addition, cervical cancer patients with high PVT-1 expression tended to have shorter overall survival than those with low PVT-1 expression. Moreover, PVT-1 overexpression promoted proliferation of Hela and C33A cells, whereas knockdown of PVT-1 induced apoptosis of Hela and C33A cells.

Conclusion: The findings of this study illustrate that increased circulating LncRNA PVT-1 is associated with poor prognosis and that PVT-1 stimulates proliferation of cervical cancer cells. These results identify an important role of PVT-1 in cervical cancer progression and a promising candidate for the prognosis and therapy of cervical cancer.
\end{abstract}

Keywords: LncRNA PVT-1; poor prognosis; apoptosis; cervical cancer

\section{INTRODUCTION}

As the leading malignancy originating from the female reproductive system, cervical cancer is one of the main causes of cancer morbidity and mortality for women around the world ${ }^{[1]}$. In 2011, more than 275,000 women diagnosed with cervical cancer died. Most of these deaths occurred in developing countries due to regional or distant metastasis ${ }^{[1,2]}$. Moreover, clinical outcomes vary significantly between patients and can be difficult to predict, and current knowledge about the

\footnotetext{
* Corresponding author: Yun-xiao Zhou. Mailing address: Department of gynaecology, the First Affiliated Hospital of Zhejiang University Medical College; Address: 79 Qingchun Rd., Hangzhou 310003, People's Republic of China.

E-mail: zhouyx2017@zju.edu.cn

Received: 15 October 2017 Accepted: 25 December 2017
}

invasion and metastasis of cervical cancer is extremely limited $[3,4]$. Therefore, finding new and effective prognostic markers and therapeutic strategies for its diagnosis and prognosis has the potential to improve treatment of cervical cancer.

Long non-coding RNAs (lncRNAs) (i.e., evolutionarily conserved non-coding RNAs that are longer than 200 nucleotides and are not translated into a protein $)^{[5,6]}$ exert regulatory functions in cellular processes, such as proliferation, apoptosis, tumorigenesis, and cancer progression and metastasis [7-9]. Recently, several studies of lncRNAs have indicated that they may play a pivotal role during the initiation and progression of human cancers, including cervical cancer ${ }^{[10-13]}$. In the present study, the researchers focused on the IncRNA PVT-1.

PVT-1 oncogene (PVT-1), which maps to chromosome $8 q 24$ (8q24) ${ }^{[14]}$, is a copy number amplification- 
associated IncRNA and exerts its influence as an lncRNA ${ }^{[15-17]}$. Amplification of $8 \mathrm{q} 24$ is one of the most frequent events in a wide variety of malignant diseases. Indeed, PVT-1 generates antiapoptotic activity in human cancers, and overexpression of PVT-1 is a powerful predictor of tumor progression and patient survival in hepatocellular carcinoma ${ }^{[10]}$; non-small cell lung cancer [18]; and colorectal ${ }^{[12]}$,ovarian, and breast cancers ${ }^{[15]}$. Furthermore, PVT-1 has been investigated as a new regulator of various biological processes, such as cell growth, apoptosis, mobility, and invasion ${ }^{[12,15] .}$ However, the association between PVT-1 and cervical cancer remains unclear.

Thus, the researchers hypothesized that circulating PVT-1 might be a potent marker for cervical cancer management. In the current study, the researchers investigated PVT-1 expression in the sera of cervical cancer patients and further explored the clinical significance and biological functions of PVT-1 in cervical cancer.

\section{MATERIALS AND METHODS}

\section{Samples collection}

The sera samples were obtained from a panel of 120 pathologically diagnosed cervical cancer patients and 118 normal, age-matched women at the First Affiated Hospital of Zhejiang University Medical College between January 2012 and December 2014. Two

Table 1 Characteristic of individuals

\begin{tabular}{lll}
\hline Variable & Patients & Normal \\
\hline Number of cases & 120 & 118 \\
Age at diagnosis (mean) & 49.5 & 48.3 \\
Clinical pathology features & & \\
WHO grading & & \\
$\quad$ I-II & 61 & \\
$\quad$ III-IV & 59 & \\
Grade & 53 \\
$\quad$ I & 42 & \\
II & 25 & \\
III & \\
Lymphatic node metastasis & 48 \\
Negative & 72 \\
$\quad$ Positive & \\
Tumor size & 49 \\
$\geq 3$ cm & 71 & \\
$<3 \mathrm{~cm}$ &
\end{tabular}

WHO: World Health Organization milliliters of whole blood were obtained before any treatment was given to the patients, and blood samples were immediately centrifuged at 3,500 rotations per minute (rpm) for 10 minutes. Subsequently, the sera samples were carefully collected and stored at $-80^{\circ} \mathrm{C}$. Clinical stage and clinicopathological factors were classified using the World Health Organization (WHO) grading and TNM system of classification, respectively. All sample data-including age, gender, lymphatic node metastasis, and tumor size-are summarized in Table 1 and were obtained from the patients' clinical and pathological records. All patients were regularly followed up on, with an observation period of 60 months. The 118 normal cases were defined as healthy ones who had neither a cervical lesion nor a history of malignancy. This study was approved by the medical ethics committee of the First People's Hospital of Hangzhou. Written informed consent was obtained from all participants.

\section{Quantitative real time PCR (qRT-PCR)}

Total RNA was extracted from sera samples using the TRIzol reagent (Invitrogen, CA, USA) according to the manufacturer's instructions. RNA was reverse transcribed using the SuperScript First Strand cDNA System (Invitrogen). The expression of PVT-1 was determined by quantitative real time PCR, which was performed on an Applied Biosystems 7500 Fast RealTime PCR system (Applied Biosystems Life Technologies, Foster City, CA, USA) and using a SYBR Premix Ex Taq II kit (Takara, Dalian City, Liaoning Province, China) according to the manufacturer's protocol. The primer sequences for PVT-1were as follows: Forward, 3'-CATCCGGCGCTCAGCT-5' and reverse, $3^{\prime}$-TCATGATGGCTGTATGTGCCA-5'. GAPDH was used to normalize mRNA expression levels. The primers were forward, 5'-GTCAACGGATTTGGTCTGTATT-3'; and reverse, 5'-AGTCTTCTGGGTGGCAGTGAT-3'. The PCR conditions were 35 cycles at $95^{\circ} \mathrm{C}$ for 30 seconds, at $95^{\circ} \mathrm{C}$ for 5 seconds, and at $60^{\circ} \mathrm{C}$ for 35 seconds. The relative level of PVT- 1 was calculated by the $2^{-\Delta \Delta \mathrm{Ct}}$ method.

\section{Cell culture}

The human cervical cancer cell lines Hela and C33A were purchased from the American Type Culture Collection (ATCC, USA) and routinely maintained in Dulbecco's modified Eagle's medium (DMEM, Gibco) with $10 \%$ fetal bovine serum (Gibco), $100 \mathrm{U} / \mathrm{ml}$ of penicillin, and $100 \mathrm{mg} / \mathrm{ml}$ of streptomycin sulfate. All cells were cultured in a humidified $5 \% \mathrm{CO}_{2}$ incubator 
at $37^{\circ} \mathrm{C}$ and used when they were in the logarithmic growth phase.

\section{Plasmid constructs and cell transfection}

The researchers used the pcDNA3.1 vector to upregulate PVT-1 expression. In summary, the resulting amplicon of PVT-1 was cloned into the pcDNA3.1 vector (Invitrogen) to generate the PVT-1-pCDNA3.1 vector. On the contrary, RNA interference was employed to down-regulate PVT-1 expression. The sequences of PVT-1-specific siRNAs were as follows: siPVT1-1, 5' - GCUUGGAGGCUGAGGAGUUT T - 3'; ; si PVT 1 - 2, $5^{\prime}$-CCCAACAGGAGGACAGCUUTT ${ }^{\prime}$. Hela and C33A cells were transfected with the PVT-1-pCDNA3.1 vector or an empty vector and siPVT1-1, siPVT1-2, or si-control using Lipofectamine 2000 (Invitrogen) according to the manufacturer's directions. Cells were incubated in a humidified air atmosphere containing $5 \% \mathrm{CO}_{2}$ at $37^{\circ} \mathrm{C}$ for 24 hours before use in assays.

\section{CCK-8 assay detects cell proliferation}

Cell proliferation was detected by the CCK-8 assay. In summary, Hela and C33A cells were seeded in a 96-well plate and transfected with PVT-1-pCDNA3.1 or empty vector pCDNA3.1. After incubation for 0 hours, 12 hours, 24 hours, and 48 hours, $10 \mu \mathrm{l}$ of CCK-8 solution (Seven Sea Biology Ltd. CO, Shanghai, China.) was added to each well, and the plate was incubated for four hours in a humidified atmosphere at $37^{\circ} \mathrm{C}$. Finally, the viability of the cells was measured at $405 \mathrm{~nm}$ using an ELISA reader (BioTek, Winooski, VT, USA) according to the manufacturer's instructions. All experiments were performed in triplicate and then the average value was calculated.

\section{Quantification of apoptosis by flow cytometry}

The cell apoptosis was detected by Annexin V-PI doublelabeling staining. In brief, an Annexin V (AV) kit and propidium iodide (PI) was purchased from Invitrogen. Hela and C33A cells were seeded in a 96-well plate and transfected with siPVT1-1, siPVT1-2, or a siPVT1 control for 24 hours. Subsequently, cells were washed twice with ice-cold phosphate-buffered saline (PBS, $\mathrm{pH}$ 7.4), re-suspended in $100 \mu \mathrm{l}$ of binding buffer, and then stained with $5 \mu$ l of Annexin V staining solution and 1 $\mu \mathrm{l}$ of PI working solution $(100 \mu \mathrm{g} / \mathrm{ml})$. The labeled cells were incubated for 15 minutes at room temperature in the dark and immediately analyzed using a FACSCalibur (Becton-Dickinson, Drive Franklin Lakes, USA) and the CellQuest software according to the manufacturer's instructions. The experiment was repeated three times.

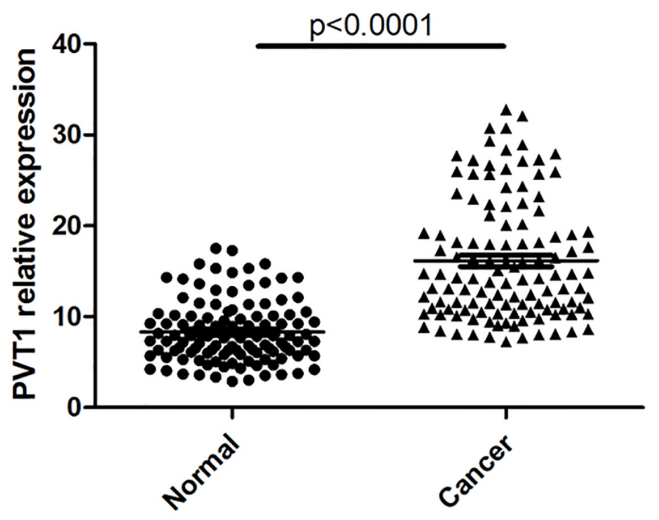

Figure 1. Circulating PVT-1 is upregulated in cervical cancer patients. qRT-PCR analyses of PVT-1expression levels in the sera of cervical cancer patients and normal controls. Results are expressed as mean \pm SD for three replicate determinations. ${ }^{* *} \mathrm{P}<$ 0.01

\section{Statistical analysis}

SPSS version 18.0 software was used for the analysis (SPSS Inc., IL, USA). All the experiments were repeated at least three times. Data were expressed as mean \pm SD. A Mann-Whitney U test was used to determine differences between the two groups, and overall survival was estimated using the Kaplan-Meier method and log-rank test. Findings were considered significant when $\mathrm{P}<0.05$ or $\mathrm{P}<0.01$.

\section{RESULTS}

LncRNA PVT-1 expression is upregulated in the sera of cervical cancer patients

Abnormal expression of PVT-1 has been found to be a prognostic indicator for human cancer patients $[10,12,15,18]$. In the current study, to investigate the clinical significance of PVT-1 in cervical cancer, the researchers first examined PVT-1 expression levels in the sera of 120 cervical cancer patients and 118 normal controls by qRT-PCR. As shown in Figure 1, compared to the normal controls, the expression of PVT-1 was significantly upregulated in the sera of cervical cancer patients $(\mathrm{P}<0.0001$, Fig. 1). The data indicated that abnormal PVT-1 expression may be related to cervical cancer pathogenesis.

Correlations between PVT-1 and clinicopathological characteristics, as well as cervical cancer patients' prognosis

Up-regulated lncRNA PVT-1 expression may serve as a new biomarker and a potential therapeutic target for several human cancers $[10,18,19]$. Therefore, the researchers further explored correlations between PVT- 
A

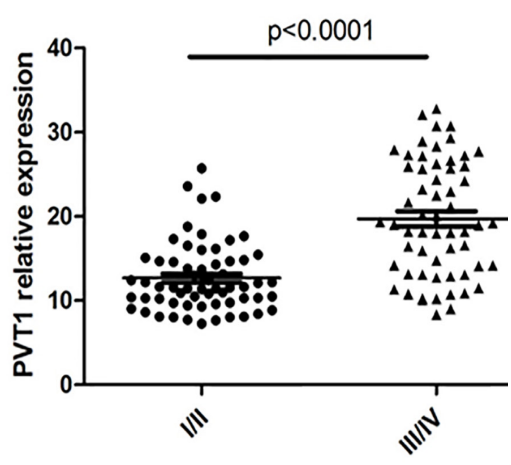

$\mathrm{C}$

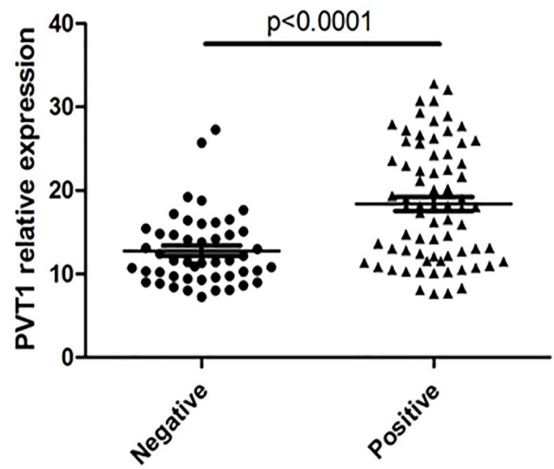

B

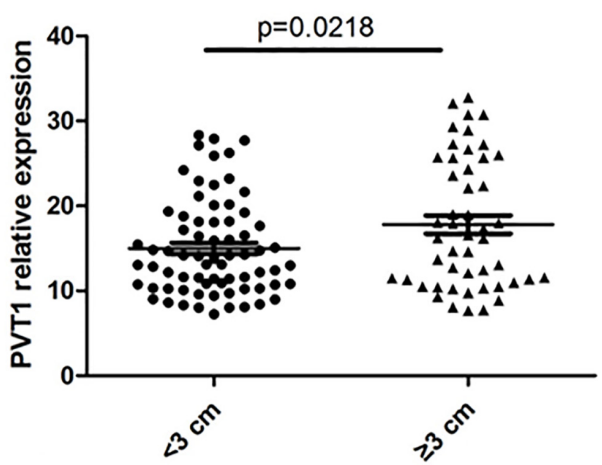

D

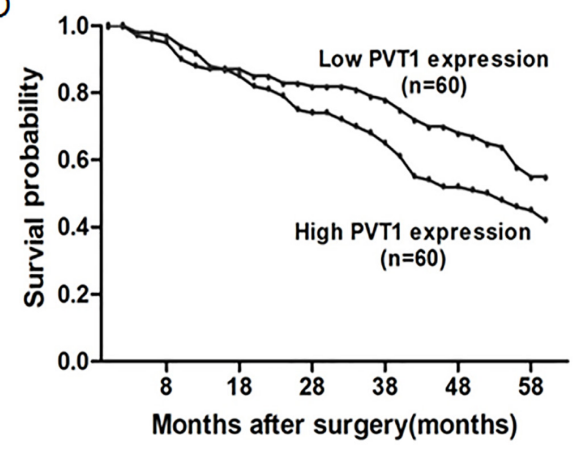

Figure 2. Correlations between circulating PVT-1 and clinicopathological characteristics, as well as patients' prognosis. PVT-1 expression was significantly higher in patients at advanced tumor stages (stages III and IV, A) and in patients whose tumor size was $\geq 3$ cm (B) and whose lymphatic node metastasis was positive (C), ${ }^{*} \mathrm{P}<0.05$. Kaplan-Meier overall survival curves for 120 patients with cervical cancer classified according to PVT-1 expression level: significantly shorter survival times for patients with high PVT-1 expression than for those with low PVT-1 expression, ${ }^{*} \mathrm{P}<0.05$ (D).

1 expression and clinicopathological characteristics. The results of the present study revealed that PVT-1 expression was much higher in patients at advanced tumor stages (stages III and IV) $(\mathrm{P}<0.0001$, Fig. 2A). To uncover the correlation between PVT-1 expression and tumor size-as well as lymphatic node metastasis-120 cervical cancer patients underwent an appropriate operation. The researchers found that PVT-1 was significantly higher in patients whose tumor size was $\geq 3 \mathrm{~cm}$ ( $\mathrm{P}=0.0218$, Fig. $2 \mathrm{~B}$ ) and whose lymphatic node metastasis was positive $(\mathrm{P}<0.0001$, Fig. 2C). These results indicate that PVT-1 expression was positively associated with WHO grading, tumor size, and lymphatic node metastasis.

To perform follow-up analysis, the 120 patients with cervical cancer were divided into a high PVT-1 expression group $(n=60)$ and a low PVT-1 expression group $(n=60)$. The association between PVT-1 expression and the survival of cervical cancer patients was investigated by Kaplan-Meier analysis and the log-rank test. As the Kaplan-Meier curve has shown, cervical cancer patients with high PVT-1 expression tend to have shorter overall survival than those with low PVT-1 expression (log-rank test, P < 0.05, Fig. 2D).
KaplanMeier analysis indicated that a high level of PVT1 was significantly related with a short period of overall survival. All these results indicate that abundant PVT1 expression is involved in poor prognosis in cervical cancer.

Up-regulation of PVT-1 promotes proliferation of Hela and C33A cells

Next, the researchers conducted PVT-1 overexpression assays using the pcDNA3.1vector to investigate the biological function of PVT-1 transcripts in cervical cancer cells. A CCK-8 assay was also performed to detect cell proliferation of Hela and C33A cells. qRT-PCR analysis showed that after transfection with PVT1-pCDNA3.1, both Hela and C33A cells showed significantly increased mRNA expression of PVT-1 compared to the pcDNA3.1 group (Fig. 3A). The results suggest that the researchers successfully upregulated PVT-1 expression in human cervical cancer cells. In addition, data from the CCK-8 assay revealed that proliferation of Hela and C33A cells was remarkably higher in PVT1-pCDNA3.1 transfected cells than in the pcDNA3.1 group (Fig. 3B, 3C). This suggests that PVT-1 overexpression significantly promoted cell 


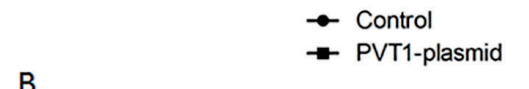

B

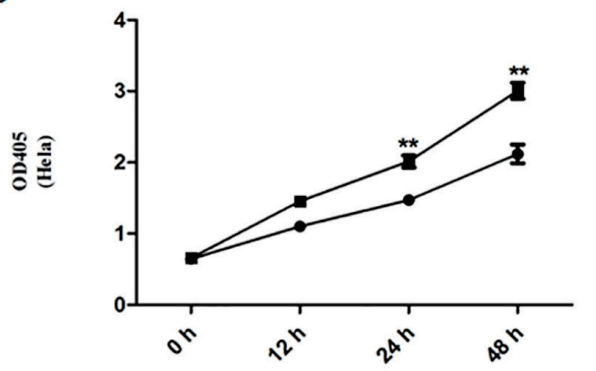

C33A

A

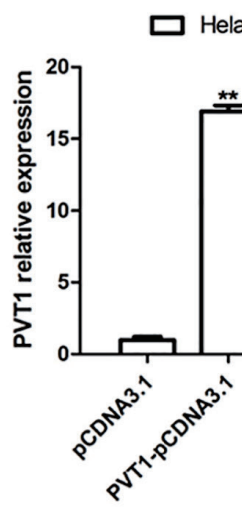

$\rightarrow$ Control

- PVT1-plasmid

C

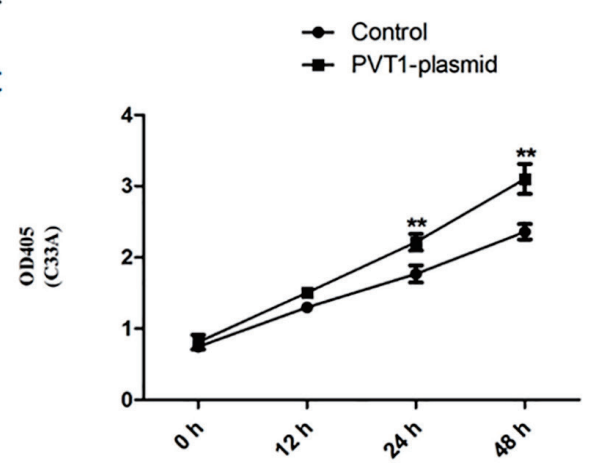

Figure 3. LncRNA PVT-1 overexpression appears to promote cell proliferation of Hela and C33A cells. Hela and C33A cells were transfected with PVT1-pCDNA3.1 or empty vector pCDNA3.1 for 24 hours, and then the expression of lncRNA PVT-1 was measured by qRT-PCR (A). Hela and C33A cells were transfected with PVT1-pCDNA3.1 or the empty vector pCDNA3.1. After transfection for 0 hours, 12 hours, 24 hours, and 48 hours, a CCK-8 assay was conducted to determine the proliferation of Hela (B) and C33A cells (C). ${ }^{* *} \mathrm{P}<0.01$.

proliferation in both cervical cancer cell lines.

Knockdown of PVT-1 appears to promote apoptosis in Hela and C33A cells

To further investigate the role of PVT-1 in human cervical cancer, PVT-1 specific siRNAs (si-PVT1-1 and si-PVT1-2) were transfected into Hela and C33A cells, respectively. Nonspecific siRNA was used as a negative control (si-control). The researchers investigated the role of PVT-1 in Hela and C33A cell apoptosis by Annexin V-PI double-labeling staining and flow cytometry. Using qRT-PCR, the researchers confirmed that PVT-1 expression in Hela and C33A cells transfected with PVT1 -specific siRNAs was significantly lower than that in cells transfected with the si-control (Fig. 4A). Moreover, flow cytometry analysis revealed that apoptosis in Hela and C33A cells was remarkably higher in si-PVT1-1 and si-PVT1-2 transfected cells than in the si-control group (Fig. 4B, 4C). These data suggest that down-regulation of PVT-1 promoted apoptosis in cervical cancer cells.

\section{DISCUSSION}

Cervical cancer is currently the second most common female cancer and remains a leading cause of cancerrelated deaths worldwide ${ }^{[20]}$. Emerging evidence has suggested that IncRNAs play oncogenic or tumor- suppressive roles in cervical cancer, including upregulated IncRNA HOTAIR expression ${ }^{[21]}$ and downregulated expression of lncRNA MALAT1 ${ }^{[22]}$,IncRNA MEG3 [23], and lncRNA GAS5 [13].Moreover, several published reports have revealed that PVT-1 contributes to cancer pathophysiology, and the human PVT-1 oncogene has been revealed to be markedly upregulated in a series of human cancers ${ }^{[15,24]}$,including colorectal ${ }^{[12]}$, ovarian, and breast cancers ${ }^{[15]}$. However, the role of lncRNA PVT-1 expression in cervical cancer and the underlying mechanism behind it is still unclear. In the current study, the clinical significance of PVT-1 was initially analyzed in the sera of 120 pathologically diagnosed cervical cancer patients and 118 normal, age-matched women. By using qRT-PCR, the research data showed that PVT-1 was significantly upregulated in cervical cancer patients (Fig. 1). Similar upregulated PVT-1 expression has been found in previous studies. An interesting study on ovarian and breast cancers indicated that PVT-1 contributes independently to ovarian and breast pathogenesis when overexpressed

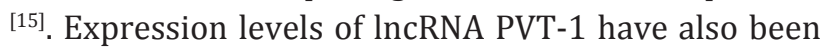
found to be significantly increased in colorectal cancer tissues ${ }^{[12]}$, hepatocellular carcinoma tissues ${ }^{[10,19]}$,lung cancer tissues and cells ${ }^{[18]}$. The high expression levels of PVT-1 in these cancers might represent a novel biomarker for predicting tumor recurrence in cancer patients and a potential therapeutic target for cancer 
A

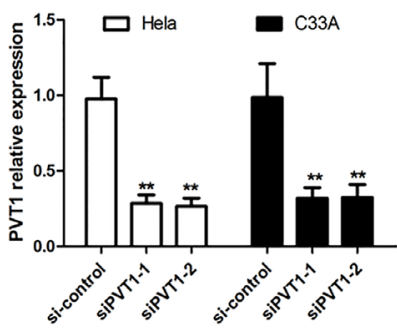

C

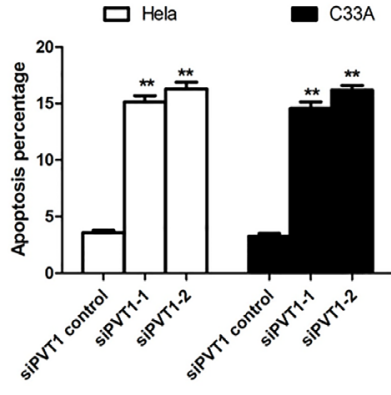

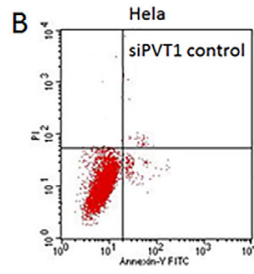
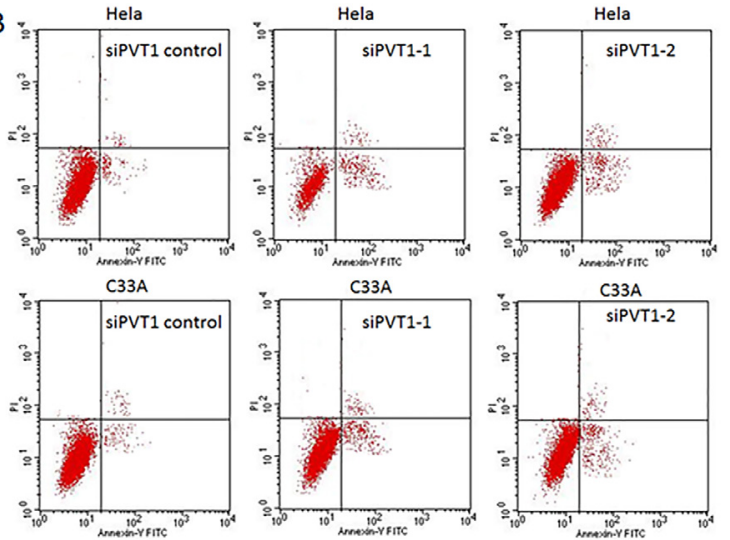

Figure 4. Knockdown of PVT-1 appears to promote apoptosis in Hela and C33A cells. qRT-PCR analysis showed that PVT-1 expression levels of both cell lines transfected with siPVT1-1 or si-PVT1-2 were significantly lower than those of si-control cells (A). Annexin V-PI double staining and flow cytometry were performed to analyze apoptosis in Hela and C33A cells. The cell apoptosis percentages of Hela and C33A cells with or without PVT-1 knockdown (B). Statistical analysis of apoptosis in Hela and C33A cells (C). ${ }^{* *} \mathrm{P}<0.01$. intervention.

Subsequently, the researchers investigated correlations between PVT-1 expression and clinicopathological characteristics. qRT-PCR analysis demonstrated that PVT-1 expression was significantly higher in patients at advanced tumor stages (stages III and IV) (Fig. 2A), as well as in patients whose tumor size was $\geq 3 \mathrm{~cm}$ (Fig. 2B) and whose lymphatic node metastasis was positive (Fig. 2C), suggesting that PVT-1 expression was positively associated with WHO grading, tumor size, and lymphatic node metastasis. A follow-up analysis was performed to further explore the association between PVT-1 expression and the survival of cervical cancer patients. KaplanMeier analysis indicated that abundant PVT-1 expression was significantly correlated with a short period of overall survival and with poor prognosis in cervical cancer (Fig. 2D). These findings are consistent with those of previous studies. Patients with high lncRNA PVT-1 expression have proven to have a significantly poorer clinical prognosis than those with low PVT-1 expression ${ }^{[12,19]}$. Moreover, the amplification of PVT-1 has been associated with reduced survival duration in several studies and has been used as an independent prognostic marker for overall survival of cancer patients $[15,18,25]$.

In the follow-up experiment, the researchers used a pcDNA3.1 vector to up-regulate PVT-1 expression and then investigate the biological function of PVT1 transcripts in two cervical cancer cell lines: Hela and C33A cells. CCK-8 assay revealed that PVT-1 overexpression appeared to significantly promote cell proliferation of Hela and C33A cells (Fig. 3B, 3C), which also supported previous research results suggesting that IncRNA PVT-1 promotes cell proliferation in hepatocellular carcinoma cells ${ }^{[19]}$ and that knockdown of PVT-1 significantly inhibits colorectal cancer cell proliferation ${ }^{[12]}$.

On the other hand, several groundbreaking studies on cell apoptosis have indicated that inhibition of PVT-1 expression also induces a strong apoptotic response in cell lines in which it is overexpressed ${ }^{[15]}$, and downregulated PVT-1 promotes colorectal cancer cell apoptosis ${ }^{[12]}$. In addition, the amplification of PVT-1 has been associated with reduced survival duration in patients treated with agents that act through apoptotic mechanisms ${ }^{[15]}$. In this study, PVT-1-specific siRNAs (siPVT1-1 and si-PVT1-2) were used to further investigate the biological functions of PVT-1in Hela and C33A cells. The results of Annexin V-PI double-labeling staining and flow cytometry revealed that forced downregulation of PVT-1 appeared to promote apoptosis in Hela and C33A cells (Fig. 4B, 4C), suggesting that PVT-1 may play an oncogenic role in cervical cancer. Additional studies are required to focus on the underlying molecular mechanism of PVT-1 in cervical cancer.

In summary, the researchers demonstrated that circulating PVT-1, which encodes an lncRNA and maps to 8q24, was commonly upregulated in cervical cancer patients and significantly correlated with advanced tumor stage, tumor size, and lymphatic node metastasis. In addition, the upregulation of PVT-1 was associated with short survival duration, suggesting that PVT-1 plays a key role in cervical cancer progression. Moreover, abnormal expression of PVT-1 appeared to exercise great influence over Hela and C33A cell proliferation and apoptosis. Thus, the findings of the present study indicate that PVT-1 is a promising prognostic indicator and therapeutic target for cervical cancer. Further study 
is required to understand the underlying mechanism of apoptosis inhibition by PVT-1.

\section{CONFLICT OF INTEREST}

The authors declare no conflict of interests.

\section{ACKNOWLEDGEMENTS}

The study was supported by the Clinical research foundation of Zhejiang Medical Association (Grant No. 2012ZYC-A10), Research project of Zhejiang population and Family planning Commission (Grant No. JSW2013-A023) and the National Natural Science Foundation of China (Grant No. 81602279).

\section{REFERENCE}

1. Siegel, R. L., Miller, K. D., and Jemal, A. (2017) Cancer Statistics, 2017. CA: a cancer journal for clinicians 67, 7-30

2. Schiffman, M., Wentzensen, N., Wacholder, S., Kinney, W., Gage, J. C., and Castle, P. E. (2011) Human papillomavirus testing in the prevention of cervical cancer. Journal of the National Cancer Institute 103, 368-383

3. Jemal, A., Bray, F., Center, M. M., Ferlay, J., Ward, E., and Forman, D. (2011) Global cancer statistics. CA: a cancer journal for clinicians $61,69-90$

4. Waggoner, S. E. (2003) Cervical cancer. Lancet $361,2217-2225$

5. Biewenga, P., van der Velden, J., Mol, B. W., Stalpers, L. J., Schilthuis, M. S., van der Steeg, J. W., Burger, M. P., and Buist, M. R. (2011) Prognostic model for survival in patients with early stage cervical cancer. Cancer 117, 768-776

6. Winkle, M., Kluiver, J. L., Diepstra, A., and van den Berg, A. (2017) Emerging roles for long noncoding RNAs in B-cell development and malignancy. Critical reviews in oncology/hematology 120, 77-85

7. Esteller, M. (2011) Non-coding RNAs in human disease. Nature reviews. Genetics 12, 861-874

8. Ponting, C. P., Oliver, P. L., and Reik, W. (2009) Evolution and functions of long noncoding RNAs. Cell 136, 629-641

9. Prensner, J. R., and Chinnaiyan, A. M. (2011) The emergence of lncRNAs in cancer biology. Cancer discovery 1, 391-407

10. Ding, C., Yang, Z., Lv, Z., Du, C., Xiao, H., Peng, C., Cheng, S., Xie, H., Zhou, L., Wu, J., and Zheng, S. (2015) Long non-coding RNA PVT1 is associated with tumor progression and predicts recurrence in hepatocellular carcinoma patients. Oncology letters 9, 955-963

11. Li, J., Wang, Y., Yu, J., Dong, R., and Qiu, H. (2015) A high level of circulating HOTAIR is associated with progression and poor prognosis of cervical cancer. Tumour biology : the journal of the International Society for Oncodevelopmental Biology and Medicine 36, 1661-1665

12. Takahashi, Y., Sawada, G., Kurashige, J., Uchi, R., Matsumura, T., Ueo, H., Takano, Y., Eguchi, H., Sudo, T., Sugimachi, K., Yamamoto, H., Doki, Y., Mori, M., and Mimori, K. (2014) Amplification of PVT-1 is involved in poor prognosis via apoptosis inhibition in colorectal cancers. British journal of cancer 110, 164-171

13. Cao, S., Liu, W., Li, F., Zhao, W., and Qin, C. (2014) Decreased expression of IncRNA GAS5 predicts a poor prognosis in cervical cancer. International journal of clinical and experimental pathology 7 , 6776-6783

14. Guttman, M., Amit, I., Garber, M., French, C., Lin, M. F., Feldser, D., Huarte, M., Zuk, O., Carey, B. W., Cassady, J. P., Cabili, M. N., Jaenisch, R., Mikkelsen, T. S., Jacks, T., Hacohen, N., Bernstein, B. E., Kellis, M., Regev, A., Rinn, J. L., and Lander, E. S. (2009) Chromatin signature reveals over a thousand highly conserved large non-coding RNAs in mammals. Nature 458, 223-227

15. Guan, Y., Kuo, W. L., Stilwell, J. L., Takano, H., Lapuk, A. V., Fridlyand, J., Mao, J. H., Yu, M., Miller, M. A., Santos, J. L., Kalloger, S. E., Carlson, J. W., Ginzinger, D. G., Celniker, S. E., Mills, G. B., Huntsman, D. G., and Gray, J. W. (2007) Amplification of PVT1 contributes to the pathophysiology of ovarian and breast cancer. Clinical cancer research : an official journal of the American Association for Cancer Research 13, 5745-5755

16. Alvarez, M. L., and DiStefano, J. K. (2011) Functional characterization of the plasmacytoma variant translocation 1 gene (PVT1) in diabetic nephropathy. PloS one 6, e18671

17. You, L., Chang, D., Du, H. Z., and Zhao, Y. P. (2011) Genome-wide screen identifies PVT1 as a regulator of Gemcitabine sensitivity in human pancreatic cancer cells. Biochemical and biophysical research communications 407, 1-6

18. Yang, Y. R., Zang, S. Z., Zhong, C. L., Li, Y. X., Zhao, S. S., and Feng, X. J. (2014) Increased expression of the lncRNA PVT1 promotes tumorigenesis in non-small cell lung cancer. International journal of clinical and experimental pathology 7, 6929-6935

19. Wang, F., Yuan, J. H., Wang, S. B., Yang, F., Yuan, S. X., Ye, C., Yang, N., Zhou, W. P., Li, W. L., Li, W., and Sun, S. H. (2014) Oncofetal long noncoding RNA PVT1 promotes proliferation and stem celllike property of hepatocellular carcinoma cells by stabilizing NOP2. Hepatology 60, 1278-1290

20. Siegel, R., Naishadham, D., and Jemal, A. (2013) Cancer statistics, 2013. CA: a cancer journal for clinicians 63, 11-30

21. Huang, L., Liao, L. M., Liu, A. W., Wu, J. B., Cheng, X. 
L., Lin, J. X., and Zheng, M. (2014) Overexpression of long noncoding RNA HOTAIR predicts a poor prognosis in patients with cervical cancer. Archives of gynecology and obstetrics 290, 717-723

22. Guo, F., Li, Y., Liu, Y., Wang, J., Li, Y., and Li, G. (2010) Inhibition of metastasis-associated lung adenocarcinoma transcript 1 in CaSki human cervical cancer cells suppresses cell proliferation and invasion. Acta biochimica et biophysica Sinica 42, 224-229

23. Qin, R., Chen, Z., Ding, Y., Hao, J., Hu, J., and Guo, F. (2013) Long non-coding RNA MEG3 inhibits the proliferation of cervical carcinoma cells through the induction of cell cycle arrest and apoptosis. Neoplasma 60, 486-492

24. Barsotti, A. M., Beckerman, R., Laptenko, O., Huppi, K., Caplen, N. J., and Prives, C. (2012) p53Dependent induction of PVT1 and miR-1204. The Journal of biological chemistry 287, 2509-2519

25. Lancaster, J. M., Dressman, H. K., Whitaker, R. S., Havrilesky, L., Gray, J., Marks, J. R., Nevins, J. R., and Berchuck, A. (2004) Gene expression patterns that characterize advanced stage serous ovarian cancers. Journal of the Society for Gynecologic Investigation 11, 51-59 